\title{
Analysis of interaction between telomeric i-motif DNA and a cyclic tetraoxazole compound
}

Shadi Sedghi Masoud, ${ }^{\ddagger[a]}$ Yudai Yamaoki, ${ }^{\ddagger[b]}$ Yue Ma, ${ }^{[a]}$ Adrien Marchand, ${ }^{[c]}$ Fernaldo Richtia Winnerdy, ${ }^{[d]}$ Valérie Gabelica, ${ }^{[c]}$ Anh Tuân Phan, ${ }^{[d]}$ Masato Katahira, ${ }^{* b]}$ Kazuo Nagasawa*a]

[a] S. Sedghi Masoud, Y. Ma, Prof. K. Nagasawa

Department of Life Science and Biotechnology, Faculty of Technology, Tokyo University of

Agriculture and Technology, Koganei, Tokyo 184-8588, Japan

E-mail:Knaga@cc.tuat.ac.jp

[b] Dr. Y. Yamaoki, Prof. M. Katahira Institute of Advanced Energy, Graduate School of Energy Science, Kyoto University, Gokasho, Uji, Kyoto 611-0011, Japan

E-mail: katahira@iae.kyoto-u.ac.jp

[c] Dr. A. Marchand, Prof. V. Gabelica

Univ. Bordeaux, INSERM, CNRS, Acides Nucléiques, Régulations Naturelle et Artificielle (ARNA,

U1212, UMR5320), IECB, 2 rue Robert Escarpit, Pessac, France

[d] Dr. F. R. Winnerdy, Prof. A. T. Phan

Division of Physics and Applied Physics, School of Physical and Mathematical Sciences, Nanyang Technological University, Singapore

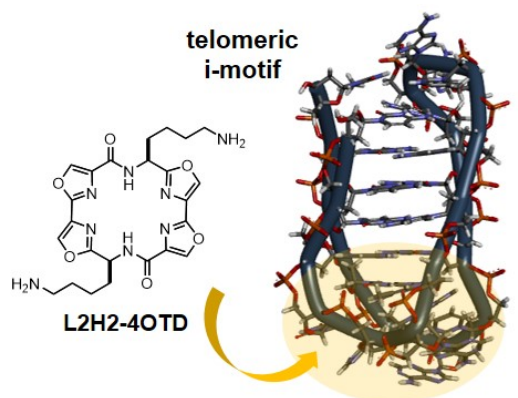

Abstract: The interaction of a macrocyclic tetraoxazole compound, L2H2-4OTD (1), bearing two aminoalkyl side chains with telomeric i-motif was investigated by means of electrophoretic mobility shift assay, circular dichroism spectroscopy, mass spectrometry and NMR analyses. The results indicate that L2H2-4OTD (1) interacts with imotif structure at two preferred binding sites. 
Non-canonical structures of DNA, so-called non-B DNA, play important roles in biological processes. For example, the G-quadruplex (G4), a non-B DNA structure found in guanine-rich sequences of telomeres and of some gene promoter regions, is involved in telomerase inhibition, transcriptional regulation, and replication. ${ }^{1}$ The stabilization of G4s by small molecules has been widely explored, both to study the biological functions of this structure, and to find candidate anticancer agents. ${ }^{2}$ The complementary sequence of a G4, thus enriched in cytosines, is known to form the i-motif structure under acidic conditions in vitro, via the interaction of hemi-protonated cytosine-cytosine base pairs (Scheme 1a and 1b). ${ }^{3}$ Although this structure was first recognized in $1993,{ }^{4}$ its biological functions are still poorly understood, partly because of the lack of selective and potent ligands. ${ }^{5}$ In the first study of a small molecule binding to i-motif in 2000 , Hurley's group examined the interaction between the telomeric i-motif and TMPyP4, ${ }^{6}$ which is a porphyrintype G4-stabilizing ligand. ${ }^{7}$ Subsequently, the same group targeted the $b c /-2$ i-motif-forming sequence with two small molecules, IMC-48 and IMC-76, and suggested for the first time that i-motif has a role in the regulation of gene expression. ${ }^{8}$

a)

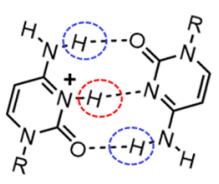

b)

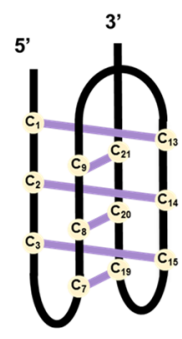

c)<smiles></smiles>

L2H2-4OTD (1) d)

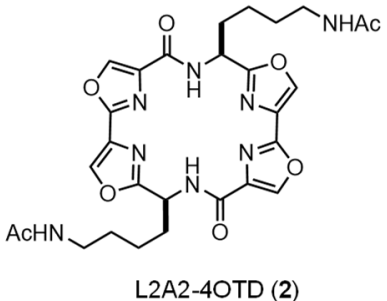

e)<smiles>CNC(CCCCCN)c1nc(-c2nc(C(=O)N[C@@H](CCCCN)c3nc(-c4nc(C(C)=O)co4)co3)co2)co1</smiles>

Scheme 1. Schematic illustrations of i-motif structure a) Cytosine-cytosine base pair structure. b) Secondary structure of telomeric i-motif structure (h-teloC) and structures of c) L2H2-4OTD (1), d) L2A2-4OTD (2), and e) Lin-L2H2-4OTD (3).

Recently, PBP $1^{9}$ and acridone derivatives ${ }^{10}$ have been developed as i-motif binders, and shown to regulate the transcriptional activities of $b c l-2$ and $c$-myc genes, respectively. However, despite the growing number of reports on i-motif-interacting agents, ${ }^{11}$ there is so far little structural information on complexes between ligands and i-motif. ${ }^{6}$ 
During our studies on G4-selective ligands, we have developed a series of cyclic polyoxazole compounds, i.e., cyclic hexaoxazoles (6OTDs) and heptaoxazoles (7OTDs), which target the G-quartet plane in G4 through $\pi-\pi$ interaction. ${ }^{12}$ In order to evaluate the influence of the size of the planar macrocycle on the G-quadruplex-stabilizing efficacy, we also synthesized penta- and tetraoxazole telomestatin analogs L2H2-5OTD and L2H2-4OTD, but found that these were less potent G-quadruplex stabilizers. ${ }^{13}$ However, because the size of the cytosine-cytosine $(\mathrm{C}-\mathrm{C})$ base pair plane in i-motif is smaller than that of the Gquartet plane in $\mathrm{G} 4$, we anticipated that the smaller macrocycles might exhibit selective i-motif-interacting ability. In this work, we confirmed the interaction of L2H2-4OTD (1) (Scheme 1c) with telomeric i-motif by electrophoretic mobility shift assay (EMSA), circular dichroism (CD) studies and electrospray mass spectrometry (ESI-MS), and we analyzed the nature of the interaction by NMR methods (chemical shift perturbation studies assisted with NOESY).

First, we examined the binding ability of L2H2-4OTD (1) to the telomeric i-motif DNA sequence h-teloC (5'-CCCTAACCC TAACCCTAACCC-3') by means of electrophoretic mobility shift assay (EMSA) on nondenaturing polyacrylamide gel with sodium cacodylate buffer $(50 \mathrm{mM}, \mathrm{pH} 5.8)$. Upon addition of $\mathrm{L} 2 \mathrm{H} 2-$ 4OTD (1), the band of the telomeric i-motif sequence is shifted due to formation of the DNA-ligand complex, and the original band completely disappears after addition of 16 equiv. of 1 (lane 8, Fig. 1a). ${ }^{14,15}$ To investigate the structural requirements of 1 for interaction with the i-motif, L2A2-4OTD (2) having N-Ac groups in the side chains and a linear analogue, Lin-L2H2-4OTD (3) were synthesized (Scheme 1d and 1e). ${ }^{16}$ Interestingly, in the cases of $\mathbf{2}$ and $\mathbf{3}$, no significant interactions were observed with h-teloC under the same conditions by EMSA (Fig. 1b and 1c). Thus, the cyclic structure and the cationic amine groups in the side chains in $\mathbf{1}$ are mandatory for interaction with the i-motif. In addition, mass spectrometric analysis indicated that two molecules of L2H2-4OTD (1) are cooperatively involved in this interaction (Fig. 2).

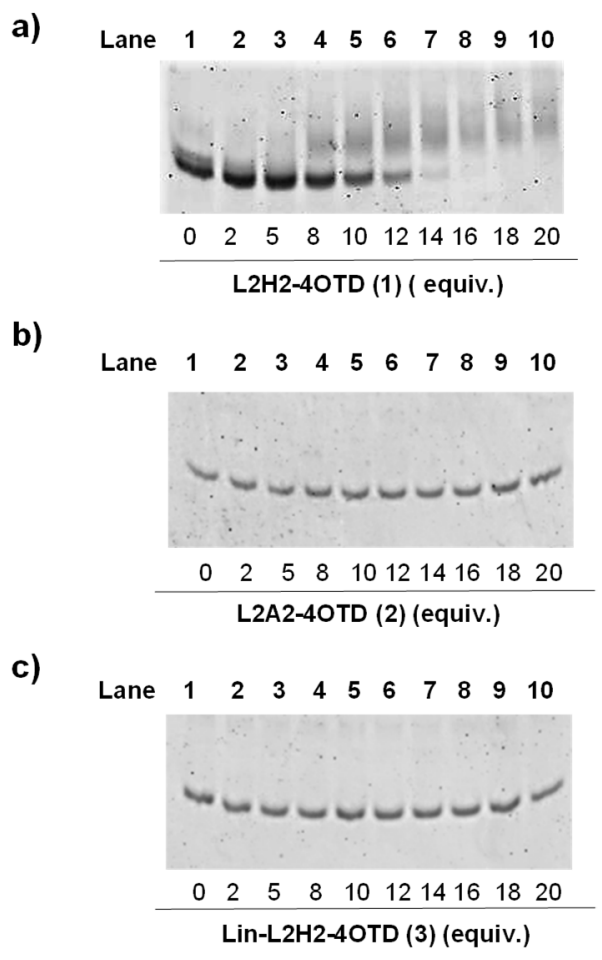

Figure 1. Electrophoretic mobility shift assay of $10 \mu \mathrm{M}$ h-teloC in $50 \mathrm{mM}$ sodium cacodylate buffer (pH 5.8) in the presence of 0 to 20 equivalents (lanes $1-10$ ) of a) L2H2-4OTD (1), b) L2A2-4OTD (2), and c) Lin-L2H2-4OTD (3). 
a)

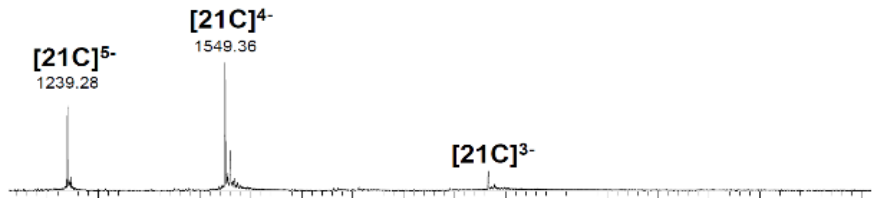

b)
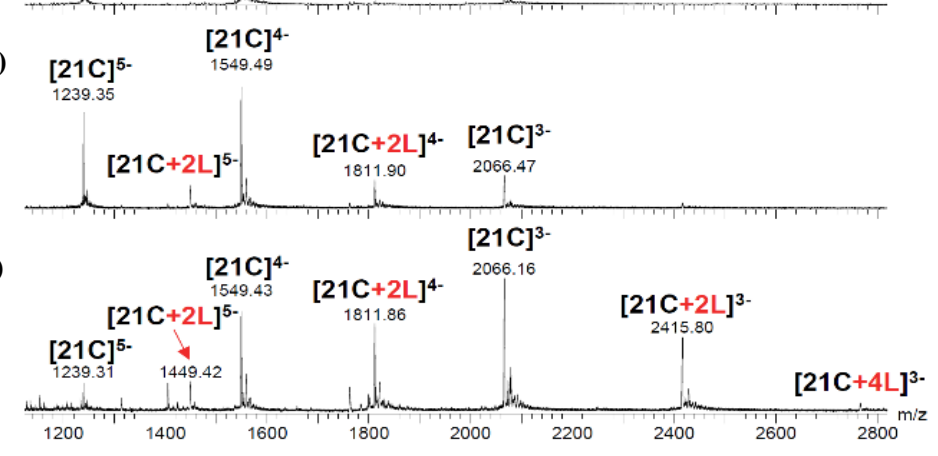

Figure 2. Electrospray mass spectra of a) $5 \mu \mathrm{M}$ telomeric i-motif (21C) with addition of b) $25 \mu \mathrm{M}$ and c) $50 \mu \mathrm{M}$ L2H2-4OTD (1) in $10 \mathrm{mM}$ TMAA buffer (pH 5.8).

Next, a CD titration experiment was carried out to investigate the effect of L2H2-4OTD (1) on telomeric i-motif structure in sodium cacodylate buffer $(50 \mathrm{mM}, \mathrm{pH} 5.8)$. The i-motif has a characteristic CD spectral signature, i.e., a positive signal at $285-290 \mathrm{~nm}$ and a negative signal near $260 \mathrm{~nm}$, related to the C-C base pair. ${ }^{17}$ The ellipticity of telomeric i-motif at $288 \mathrm{~nm}$ decreases and slightly shifts towards lower wavelength upon addition of L2H2-4OTD (1) (Fig. 3), suggesting that binding of 1 induces structural changes. ${ }^{18}$

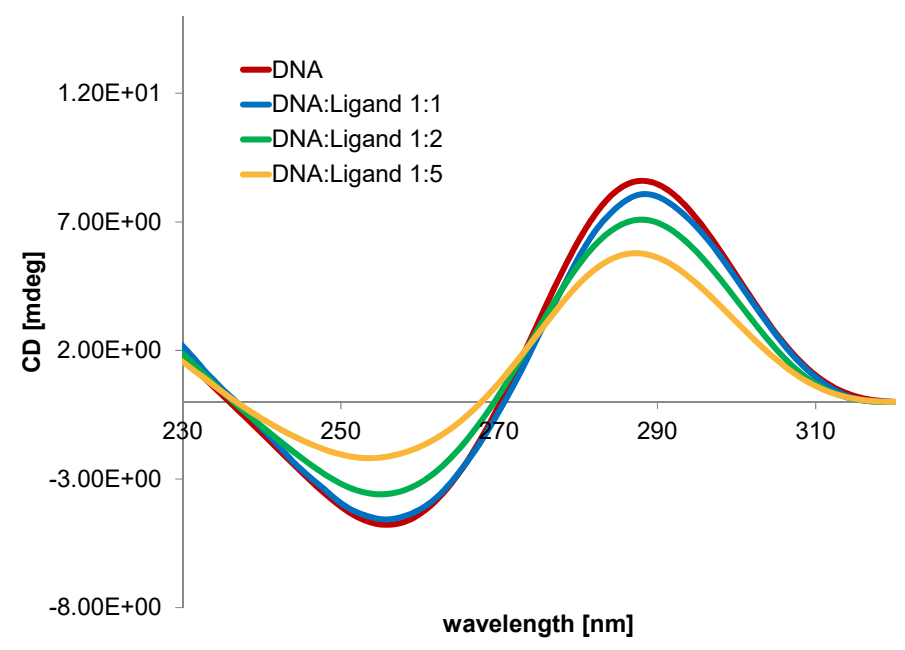

Figure 3. CD spectral change of $10 \mu \mathrm{M}$ h-teloC upon titration with L2H2-4OTD (1) in $50 \mathrm{mM}$ sodium cacodylate buffer ( $\mathrm{pH}$ 5.8). 
To gain further insight into the structural effect of L2H2-4OTD (1) on the telomeric i-motif DNA, NMR analyses were carried out using h-telo ${ }^{\mathrm{m}} \mathrm{C}\left(5^{\prime}-\mathrm{CCCTA}{ }^{5 \mathrm{~m}}\right.$ CCCTAACCCUAA CCCT-3'), an analogue of $\mathrm{h}$-teloC. This sequence is previously studied by NMR, and reported to form a homogenous i-motif structure under acidic conditions, as schematically illustrated in Fig. 4c; the reported assignments were utilized in this study. ${ }^{19,20}$

The imino proton spectra of h-telo ${ }^{\mathrm{m} C}$ during titration with L2H2-4OTD (1) are shown in Fig. 4a. Upon addition of up to one equivalent of L2H2-4OTD (1), the imino proton signal of the C3-C15 base pair is shifted toward higher field, whereas the signals of the imino protons of the $\mathrm{C} 2-\mathrm{C} 14$ and $\mathrm{C} 8-\mathrm{C} 20$ base pairs are not significantly shifted (Fig. $4 a$ and $b$ ). These observations suggest that the preferred binding site of L2H2-4OTD (1) is close to the C3-C15 base pair, and the chemical shift of the corresponding imino proton signal being perturbed by this binding.
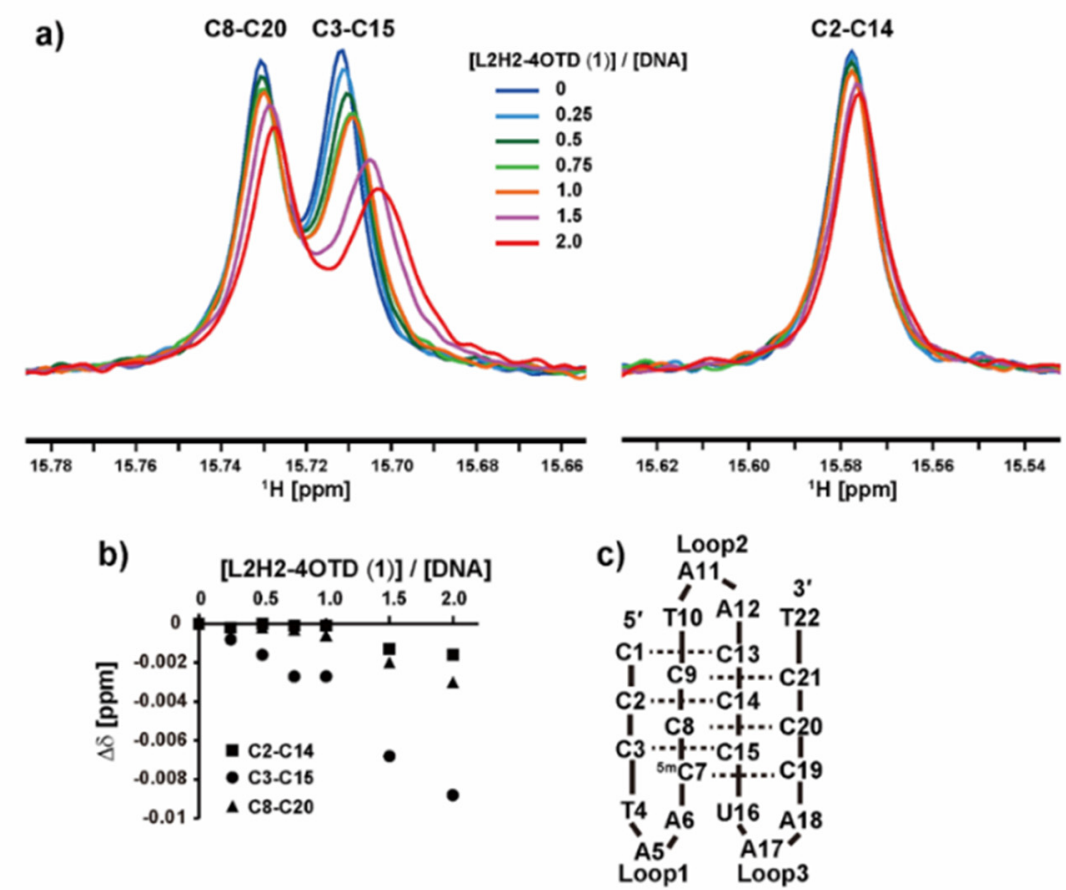

Figure 4. Chemical shift perturbation (CSP) of the imino proton signals of C-C base pairs of 100 $\mu \mathrm{M}$ h-telo ${ }^{\mathrm{m} C}$ during addition of L2H2-4OTD (1) in $50 \mathrm{mM}$ sodium cacodylate buffer $(\mathrm{pH} 5.8)$ at 25 ${ }^{\circ} \mathrm{C}$. a) The imino proton spectra. The [L2H2-4OTD (1)] / [h-telo ${ }^{\mathrm{m}} \mathrm{C}$ ] ratio was $0,0.25,0.5,0.75,1.0$, 1.5 or 2.0, as indicated. b) CSP values of the imino proton signals. c) Schematic illustration of the i-motif structure of $\mathrm{h}$-telo ${ }^{\mathrm{m}} \mathrm{C}$. The cytosine-cytosine base pairs are indicated by dotted lines.

The upfield shift of the imino proton signal may imply that the binding of L2H2-4OTD (1) to h-telom C slightly distorts the $\mathrm{C} 3-\mathrm{C} 15$ base pair, which may allow chemical exchange of the imino proton with water. On the other hand, the unchanged chemical shift of the imino proton signals of the C2-C14 and C8-C20 base pairs indicates that these $\mathrm{C}-\mathrm{C}$ base pairs are unaffected, and the original i-motif structure may be retained with up to an equimolar amount of 1 . However, when more L2H2-4OTD (1) is added, the imino proton signals of the $\mathrm{C} 2-\mathrm{C} 14$ and $\mathrm{C} 8-\mathrm{C} 20$ base pairs start to shift towards higher field as well (Fig. 4a and b). This suggests that the secondly preferred binding site of L2H2-4OTD (1) is close to the C2-C14 and C8C20 base pairs, and this interaction causes perturbation and slight distortion on these base pairs. This idea is consistent with the $C D$ titration results. 
To investigate the binding site of L2H2-4OTD (1) in the telomeric i-motif structure more precisely, we carried out chemical shift perturbation (CSP) analysis of non-exchangeable proton signals. First, we recorded the NOESY spectra of h-telo ${ }^{\mathrm{m}} \mathrm{C}$ both in the absence and presence of equimolar L2H2-4OTD (1) (Fig. S5). Signals in the presence of L2H2-4OTD (1) were assigned on the basis of the reported assignments of non-exchangeable protons. ${ }^{19}$ Then, CSP caused by the addition of L2H2-4OTD (1) was calculated for the assigned signals. The results are shown in Fig. 5. Three of the four largest CSPs were observed for the A5, A6 and T4 residues (Fig. 5a), which are located at Loop 1 of the i-motif structure (Fig. 4c). CSPs larger than the average were observed for residues located in Loop 1, Loop 3 and the region close to these loops, except for $\mathrm{A} 12 \mathrm{H} 1{ }^{\prime}$ and $\mathrm{C} 1 \mathrm{H} 5$ (Fig. 5a and b).

a)
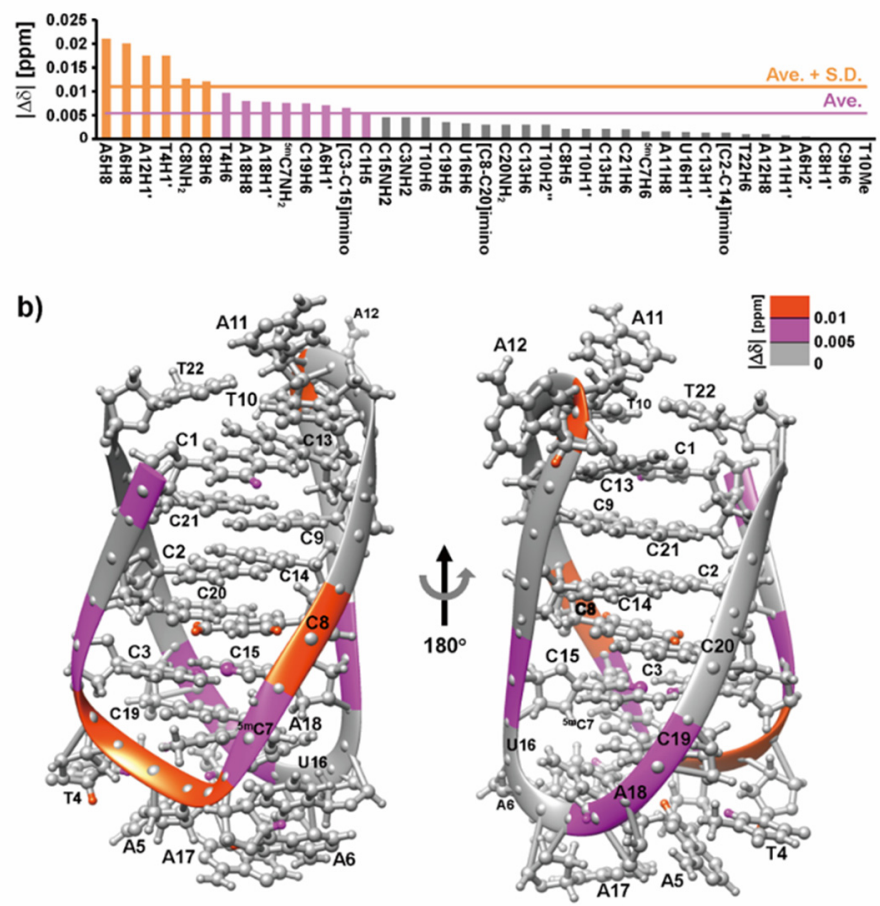

Figure 5. CSP of non-exchangeable proton signals of $400 \mu \mathrm{M}$ h-telo $\mathrm{m} C$ upon addition of equimolar L2H2-4OTD (1). a) Absolute values of CSP. Ave. and S.D. mean the average and the standard deviation of absolute values of CSP, respectively. CSPs larger than Ave. $(0.005 \mathrm{ppm})$ are colored magenta and those larger than Ave. plus S.D. (0.01 ppm) are colored orange. b) Mapping of CSP on the i-motif structure of h-telo ${ }^{m} \mathrm{C}$ (PDB ID: 1EL2) drawn as a ball and stick model with ribbon representation.

This suggests that the most preferred binding site of L2H2-4OTD (1) is comprised of Loop 1 and Loop 3 of i-motif structure and their neighbouring region. Thus, it is reasonable that addition of equimolar $\mathrm{L} 2 \mathrm{H} 2-$ 4OTD (1) to h-telo ${ }^{\mathrm{m}} \mathrm{C}$ induces CSP only for the imino proton of the C3-C15 base pair, which is located close to the most preferred binding site.

In conclusion, we have characterized L2H2-4OTD (1) as a ligand of the telomeric i-motif. Our results show that two molecules of L2H2-4OTD (1) bind cooperatively to the i-motif structure. NMR analysis indicated that the most preferred binding site is comprised of Loop 1 and Loop 3 of telomeric i-motif and their neighboring region and that binding of L2H2-4OTD (1) to this site causes slight distortion of the C3C15 base pair. When the second couple of L2H2-4OTD (1) binds to the secondly preferred binding site, the C2-C14 and C8-C20 base pairs are affected as well. 


\section{Experimental Section}

Experimental Details can be found as supporting information.

\section{Acknowledgements}

SS is grateful for support from Rotary Yoneyama Memorial Foundation Scholarship (YD). YM is grateful for financial support in the form of JSPS Predoctoral Fellowships for Young Scientists. VG and AM were supported by Inserm (ATIPAvenir Grant no. R12086GS), Conseil Regional Aquitaine (Grant no. 20121304005), and the EU (FP7-PEOPLE-2012-CIG-333611 and ERC-2013-CoG616551-DNAFOLDIMS). MK and $Y Y$ are grateful for partial support from JSPS KAKENHI (16K14678, 18H04550, 18K19397 and 17K14515). ATP was supported by grants from Nanyang Technological University. This work was supported in part by Grants-in-Aid for Scientific Research (B) from JSPS (23310158 and 26282214) and a Grant-in-Aid for Challenging Exploratory Research from JSPS (26560444). This research was also partially supported by a grant from Japan Agency for Medical Research and Development (AMED, 16cm0106102h0001).

Keywords: i-motif • ligand $\bullet$ macrocyclic $\bullet$ tetraoxazole $\cdot$ NMR

[1] a) M.L. Bochman, K. Paeschke, V.A. Zakian, Nat. Rev. Genet. 2012, 13, 770-780. b) A. Verma, V. K. Yadav, R. Basundra, A. Kumar, S. Chowdhury, Nucleic Acids Res. 2009, 37, 4194-4204. c) A. SiddiquiJain, C. L. Grand, D. J. Bearss, L. H. Hurley, Proc. Natl. Acad. Sci. U. S. A. 2002, 99, 11593-11598. d) Y. Kanoh, S. Matsumoto, R. Fukatsu, N. Kakusho, N. Kono, C. Renard-Guillet, K. Masuda, K. lida, K. Nagasawa, K. Shirahige, H. Masai, Nat. Struct. Mol. Biol. 2015, 11, 889-897.

[2] a) S. Neidle, FEBS. J. 2010, 277, 1118-1125. b) S. Balasubramanian, L.

H. Hurley, S. Neidle, Nat. Rev. Drug. Discov. 2011, 10, 261-275.

[3] a) A. T. Phan, J. L. Mergny, Nucleic Acids Res. 2002, 21, 4618-4625. b)

H. A. Day, P. Pavlou, Z. A. E. Waller, Bioorg. Med. Chem. 2014, 22, 4407-4418. c) S. Benabou, A. Aviñó, R. Eritja, C. González, R. Gargallo, RSC, Adv. 2014, 4, 26956-26980.

[4] K. Gehring, J. L. Leroy, M. Gueron, Nature. 1993, 363, 561-565.

[5] Recently, the possibility of i-motif formation in nuclei of human cells was suggested, based on the introduction an antibody fragment that selectively recognizes this structure. M. Zeraati, D. B. Langley, P. Schofield, A. L. Moye, R. Rouet, W. E. Hughes, T. M. Bryan, M. E. Dinger, D. Christ, Nature Chemistry. 2018, 10, 631-637.

[6] O. Y. Fedoroff, A. Rangan, V. Chemeris, L. H. Hurley, Biochemistry. 2000, 39, 15083-15090.

[7] R. T. Wheelhouse, D. Sun, H. Han, F.X. Han, L. H. Hurley, J. Am. Soc. 1998, 120, 3261-3262 .

[8] S. Kendrick, H-J. Kang, M. P. Alam, M. M. Madathil, P. Agrawal, V. Gokhale, D. Yang, S. M. Hecht, L. H. Hurley, J. Am. Chem. Soc. 2014, 136, 4161-4171.

[9] M. Debnath, S. Gosh, A. Chauhan, R. Paul, K. Bhattacharyya, J. Dash, Chem. Sci. 2017, 8, 74487456.

[10] B. Shu, J. Cao, G. Kuang, J. Qiu, M. Zhang, Y. Zhang, M. Wang, X. Li, S. Kang, T.-M. Ou, J.-H. Tan, Z.-S. Huanga, D. Li, Chem. Commun. 2018, 54, 2036-2039.

[11] a) E. P. Wright, H. A. Day, A. M. Ibrahim, J. Kumar, L. J. Boswell, C. Huguin, C. E. Stevenson, K. Pors, Z. A. Waller, Sci. Rep. 2016, 6, 39456. b) H. A. Day, P. Pavlou, Z. A. E. Waller, Bioorg. Med. Chem. 2014, 16, 4407-4418. c) Q. Sheng, J. C. Neaverson, T. Mahmoud, C. E. M. Stevenson, S. E. Matthews, Z. A. E. Waller, Org. Biomol. Chem. 2017, 27, 5669-5673. d) H.-J. Kang, S. Kendrick, S. M. Hecht, L. H. Hurley, J. Am. Chem. Soc. 2014, 136, 4172-4185.

[12] a) K. lida, K. Nagasawa, Chem. Rec. 2013, 13, 539-548. b) X.J. Chung, B. Heddi, M. Tera, K. lida, K. Nagasawa, A. T. Phan, J. Am. Chem. Soc. 2013, 135, 13495-13501. c) M. Tera, H. Ishizuka, M. Takagi, M. Suganuma, K. Shin-ya, K. Nagasawa, Angew. Chem., Int. Ed. 2008, 47, 5557-5560.

[13] S. Sedghi Masoud, Y. Tsushima, K. lida, K. Nagasawa, Heterocycles. 2015, 90, 874-882.

[14] This interaction also investigated using other sequences (Fig. S3).

[15] For oligonucleotides used in experiments see Table S1.

[16] L2H2-4OTD (1), L2A2-4OTD (2) and Lin-L2H2-4OTD (3) were synthesized based upon our previously reported protocol (Scheme S1 and S2). 
[17] a) M. Vorlickova, I. Kejnovska, K. Bednarova, D. Renciuk, J. Kypr, Chirality, 2012, 24, 691-698. b) J. Kypr, I. Kejnovska, D. Renciuk, M. Vorlickova, Nucleic Acids Res. 2009, 37, 1713-1725.

[18] Similar behaviour was observed with the i-motif-forming sequences of $c-k i t$ and $b c l-2$ upon titration with L2H2-4OTD (1) (Fig. S4 a and b).

[19] A. T. Phan, M. Gueron, J. L. Leroy, J. Mol. Biol. 2000, 26, 123-144.

[20] A CD titration experiment was carried out with h-telom $\mathrm{C}$ (Fig. S4 c). 